Brit. J. industr. Med., 1952, 9, 108.

\title{
THE MECHANISM OF DISPERSION OF COAL PARTICLES IN THE LUNGS OF MINERS
}

\author{
BY \\ A. POLICARD \\ From the Centre d'Études et de Recherches des Charbonnages de France, Paris
}

(RECEIVED FOR PUBLICATION JANUARY 15, 1952)

Coal particles inhaled by miners in the course of their work, especially those of 2 microns in diameter and less, are carried as far as the alveoli. Particles which have reached the alveoli and become attached to their surface are phagocytosed by the alveolar cells, and in this way dust cells are formed. This is the first stage in the migration of coal-dust, of which the second is its deposition in the periarterial and peribronchial connective tissue sheaths, as well as in certain other fibrous tissues such as the pleura. Some particles can also travel even further, into the tracheo-bronchial glands and beyond, but these will not be considered here.

This paper is concerned with the histological and physiological mechanisms of dust transportation and the pathways along which dust cells travel. It is a particularly complicated problem, because animal experiments cannot give us reliable information as the behaviour of the lungs is different in each species. The lungs of a rabbit do not behave in exactly the same way as those of a rat, still less as those of a man. Extrapolation from animal species to man is therefore not valid, as I have emphasized elsewhere (Policard, 1950). I need not go further into the question here. The only acceptable observations, therefore, are those made on man himself but there other difficulties arise, of which the most serious is that when the thorax is opened for removal of the lungs, they collapse. This collapse of the lung, which is accentuated by the action of fixatives, upsets normal relationships. The contraction does not produce lesions but changes the topographical relationship of the structures. It would be best to fix the lungs in position without opening the thorax, but although this is theoretically possible, it would not be practicable in the case of man. It is possible, however, to inflate the lungs before fixing them, although it is difficult under the conditions of ordinary hospital necropsies. Moreover, this procedure does not usually give good results because of the postmortem fragility of the alveolar walls, which rupture on inflation. Under normal post-mortem conditions, one can only obtain a lung which is not collapsed when the alveoli of part of the lung are filled with a transudate, as in a case of acute oedema of the lung, or blood, as in the case of pulmonary infarct ; for then, this part of the lung does not collapse when the thorax is opened and the alveoli are still in their normal position of distention even after fixation. It is a case of this kind which constitutes the basis of this paper.

\section{Case Report}

A miner aged 40, who had worked in coal for 20 years and was in excellent health, was caught while at work in coal dust by an escape of fire-damp (methane) and collapsed with asphyxia. (Methane has no toxic effect on pulmonary tissue but kills by simple asphyxia.) The miner was removed unconscious after some minutes and taken to the surface. Shortly afterwards he showed signs of acute pulmonary oedema (anoxic oedema) and died two hours after the accident. For medico-legal reasons the necropsy was carried out five hours after death, that is seven hours after the beginning of asphyxia. The lung showed the usual appearance of acute oedema and in particular it showed no collapse.

The interest of such a case, as far as it concerns our problem, is (1) that the man was struck down suddenly while at work and while actually breathing coal dust. (2) The additional process of passive oedema prevented the collapse of the lung that usually occurs after death and so avoided displacement of pulmonary structures. (3) The tissues were fixed a relatively short time after death and so allowed histological examination to be carried out under good conditions.

A histological study of this case has shown the usual appearances seen in coalworkers' lungs such as have been described by Gough (1947) and by Heppleston (1951) : the presence of an accumulation 
of dust cells in the peri-arterial and peribronchial tissues, which are hypertrophied and fibrosed, though not to a very great extent (these accumulations constitute the macules of the Welsh workers), and clear evidence of a certain amount of focal emphysema of the type described by Gough (1940, 1947). In this lung the following histological observations could be made.

\section{Dust in the Alveoli Phagocytosed by Alveolar Cells}

In the alveolar cavities, well dilated by the presence of the oedematous exudate, there were practically no free particles but, on the other hand, numerous alveolar cells stuffed with coal particles, the cells being free in the transudate or attached to the alveolar wall. The rarity of free particles can be easily understood. The transudation of blood plasma through the capillaries, resulting from the development of oedema, has probably carried away the particles which were on the alveolar walls. These particles could also have been phagocytosed during the period of formation of oedema, the exudate constituting a kind of culture medium in which the phagocytes could operate. However it may be, these observations do not allow us to draw any conclusion about the presence of free particles in the alveoli ; the data are only applicable to dust cells.

The dust cells found in the alveoli were quite typical. They were rounded or ovoid structures, $18-20 \mu$ in diameter, with clearly defined borders but no sign of a membrane. They contained an ovoid or kidney-shaped nucleus, 6-8 $\mu$ in diameter, with a fine chromatin network. This arrangement is well known.

The arrangement of the coal particles was variable and two chief types of cells could be distinguished. In some the particles, which were few, were well spaced; sometimes there were two or three to the cell, sometimes 10 to 20 . They were situated in the cytoplasm, generally close up against the nucleus. The latter was not modified by their presence. No cells were to be seen in the periphery of the cytoplasm. In the other type of cell the particles were closely packed and were situated in the centre of the cell in contact with the nucleus.

Observations on man differ somewhat from those made in animal experiments. In the latter the cells always enclose a very large number of particles, never two or three. This suggests that isolated particles are not phagocytosed by the cell, which only picks up aggregates of particles formed outside it and ingests them en bloc. This process, often clear in the animal, does not seem to be the only one, phagocytosis of single particles being also possible. This is a question which requires further study.
The particles seen were entirely carbonaceous. In polarized light practically no birefringent particles were seen. They were all black or brown and composed of coal. Most of them were between 0.4 and $0.8 \mu$ in diameter. Particles from 1 to $2 \mu$ were much less frequent and only occasionally particles from 3 to $8 \mu$ were seen.

The dust cells were usually free in the liquid which filled the alveolar cavities. A few, however, were still attached to the alveolar walls. Besides these dust cells one occasionally found alveolar cells of the same type but containing no dust. No dust cells were seen showing signs of degeneration and cytolysis, that is to say distended and more or less disrupted.

Dust cells so formed in the alveoli pass into the interstitial tissue and then take several courses. Two points must be considered here ; the route by which they are removed, and the mechanism by which it is carried out, whether passive translation by the current of lymph or active amoeboid movement. It is obvious that as no direct demonstration of these movements was possible, any answer to these questions can only be indirect deductions from morphological observations, and they are correspondingly uncertain.

Apart from those dust cells which reach the bronchioles, and hence the bronchi and trachea, one must consider two chief routes of penetration into the interstitial tissue, and especially into the peri-arterial and peribronchial sheaths ; the route through the terminal bronchioles and the route through the periphery of the sheaths.

\section{Penetration of Dust Cells at the Level of the Terminal Bronchioles}

At the end of the terminal bronchioles there is a point of transition to the alveolar duct, a position of histological and physiological importance (Policard and Collet, 1951). At this level the bronchial epithelium ceases and the connective tissue of the bronchial sheath is in contact with the alveolar air and is not covered with epithelium. Dust cells coming from the alveoli are carried towards the bronchi, in the thin layer of liquid which covers the surface of this region. At the point where the bronchial epithelium begins, the cells can take one of two routes. They can pass over the epithelium and so reach the bronchi and be removed by expectoration, or they can pass under the epithelium and embed themselves in the connective tissue between its collagen fibres. In this way these cells, whether of their own accord or translated passively by the current of lymph, are carried to the peribronchial interstitial tissue. The dust cells can also attain the adjacent peri-arterial connective tissue, 
-with which the latter tissue is always continuous. By their accumulation at certain points, the dust cells form the peribronchial and peri-arterial nodules which can be seen in lung sections in coalworkers' pneumoconiosis.

The mechanism by which particles penetrate to the end of the bronchioles is only a particular case of a general process. It also occurs with microorganisms, as has been known for a long time.

The same movement of dust cells has often been observed in animal experiments. On the other hand, it is very difficult to observe it in man because of the disturbance of the lung produced by postmortem collapse. In the case we have studied it was easily observed.

Penetration of Dust Cells into Perivascular and Peribronchial Tissue by the Peripheral Route

Dust cells in the alveoli can also get into the periarterial and peribronchial sheaths from their periphery. The mechanism seems to be as follows. The peri-arterial and peribronchial sheaths are surrounded by alveoli. At this level, as in all alveoli, this sheath is in contact with the alveolar surface. There is no continuous epithelium at this level but only a very incomplete layer of alveolar cells capable of phagocytosis. By their own movement, and also because of the flow of lymph from their surface towards the bronchi, dust cells can penetrate into the spaces between the fibres of the perivascular and peribronchial sheaths.

The following points should be noted. Dust cells can penetrate into the sheath from the periphery at all points but not always with equal ease. There are points where penetration is quite easy and there one finds a large number of cells. This probably explains the irregular form of the anthracotic nodule in the lungs of coal miners. The reason for this difference is still unknown but it seems possible that it depends on whether the fibrous tissue is closely or loosely packed.

Many anthracotic nodules, in fact most, are surrounded by emphysematous alveoli (focal emphysema of Gough). The presence of this emphysema does not seem greatly to affect the penetration of dust cells at these points. Often around certain partially developed anthracotic nodules, alveoli can be seen in a state of collapse. This condition seems to favour peripheral growth of the nodule. The alveolar walls collapse against the sheaths, increasing their diameter. In becoming attached and more or less fused to them, the alveolar walls enclose the dust cells which lie inside the alveolar cavities. This method of growth of anthracotic nodules seems to be common and important and is one which has been generally neglected. It is not seen in animal experiments but is very clearly seen in man under favourable conditions.

Often an accumulation of dust cells is seen in the lymph channels as numerous as in the connective tissue and, as a result of this accumulation, there is lymphatic obstruction. This suggestion, although fairly well founded, calls for certain reservations. In many places dust cells are found outside but not within the connective tissue lymphatics. This is particularly well seen in an undeveloped anthracotic nodule. The central artery shows a loose layer of connective tissue which isolates it from the pulmonary tissue (mobile layer of Von Hayek) and which contains the lymphatics. In the large nodules, on the other hand, the layer of dust cells extends up to the media of the artery. In certain cases also, in the most advanced stages of pneumoconiosis, dust cells may be seen in the media itself, which in this event is always abnormal. This suggests that the dust cells first arrive at the periphery of the sheaths. Particles which come via the lymphatics are carried by the lymph stream beyond the lung into the tracheo-bronchial glands. Interference with lymphatic function is a late phenomenon. Only then do the dust cells begin to accumulate in the loose inner layer of the connective tissue sheath.

These observations are of interest in so far as they concern the origin of vascular disturbances which develop in advanced coalworkers' pneumoconiosis.

\section{Characteristics of Coal-dust Cells in Anthracotic Nodules}

When they are free in the alveoli, coal-dust cells are regular spherical or ovoid structures but when they are in the fibrous tissue sheaths they have a different appearance. They are very irregular and are distorted by the surrounding connective tissue fibres. They behave like all tissue cells, whether fibroblasts or histiocytes, their shape depending on the more resistant neighbouring structures. In accordance with the arrangement of these surrounding structures, the dust cells are elongated or flattened but relatively much less than normal connective tissue cells which do not contain particles. The presence of hard particles prevents the cells from being deformed beyond a certain limit. Moreover, in cross section, the sheaths themselves are star-shaped.

In anthracotic nodules the cells are cytologically much altered. They have degenerated and their nucleus has usually disappeared. Their cytoplasm is hardened into a sort of cement (Policard and Collet, 1951) which encloses the particles and keeps them aggregated. This degeneration occurs by a fairly characteristic kind of dessication. One never 
sees coal-dust cells distended or disrupted by proteolysis. This suggests that, by a mechanism as yet unknown, the coal particles inhibit the proteolytic enzymes normally present in the cells.

\section{Fate of Collapsed Alveoli at the Periphery of Anthra- cotic Nodules}

The walls of the collapsed alveoli surrounding the sheaths and the anthracotic nodules are joined together and seem to fuse to one another to form a fibrous mass. The reality of this fusion appears to be undoubted but its mechanism remains obscure. The following hypothesis is possible. The collapse of the alveoli leads to circulatory disturbances with the formation of an exudate into what remains of the alveolar cavities, thus distending the alveolar walls. This produces some repair of the connective tissue of the walls with a formation of new collagen ; initially, as usual, by precollagenous or reticulin fibres. Little by little the reticulin fibres are converted, as is customary, into true collagen fibres.

The part of alveolar collapse in the fibrous thickening of the sheaths from the outside involves the role (often discussed in pulmonary pathology) of atelectasis in the genesis of certain pulmonary fibroses.

\section{The Passage of Single Particles in the Alveoli into the Peri-arterial Sheaths}

It may be asked whether coal particles can be carried singly by the lymph and so reach the sheaths without being carried by cells. Once in the sheaths they could be phagocytosed by histiocytes present at that point.

In the course of the present investigation nothing has been seen which leads to a belief in the existence of such a process. In the peri-arterial sheaths particles are always collected in cells in a mass of the shape of the dust cell. It is true that it is important not to consider as particles of independent origin those which have clearly been displaced by the action of the microtome knife. This cause of error is well known but it is not always easy to avoid. On the other hand, in the sheaths in their normal state sufficient histiocytes are not found to explain the presence in this region of the large number of dust cells which are found there. Without actually denying the possibility of the transport of free particles from the alveoli to the sheaths, it seems it must be admitted that if this process exists it is most exceptional.

\section{Conclusions}

With the reservations which are necessary when making physiological deductions from histological observations, it seems that it may be suggested that the anthracotic nodule so characteristic of the lung of coal miners could be produced in the following way.

Two processes are involved : a proportion of the dust cells formed in the alveoli reach the periarterial and peribronchial connective tissue by two different routes. Some dust cells enter the pulmonary connective tissue at the level of the terminal bronchioles and reach the lymphatic channels normally present in the sheaths. So long as the sheaths are not abnormal, the dust cells are carried by the lymph and pass to the tracheo-bronchial glands. Other dust cells formed in the alveoli adjacent to the sheaths penetrate them from outside. They are found in the periphery of the sheaths. It seems that the dust cells remain stationary and are not carried to the lymph nodes.

The anthracotic nodule appears to result from the growth of sheaths thickened in the following way. At the periphery of the sheaths the alveoli collapse. Their walls are applied to the external surface of the sheaths which is thickened by them. The dust cells which happen to be in the alveolar cavities are thus included in the thickened wall of the sheaths, that is to say, in the outer part of the anthracotic nodule. The dust cells thus included in the nodule seem destined to remain permanently fixed there.

In the sheaths which have been progressively thickened and changed into anthracotic nodules some changes in the connective tissue occur, a process which is being actively studied at present.

\section{REFERENCES}

Gough, J. (1940). J. Path. Bact., 51, 277. (1947). Occup. Med., 4, 86.

Heppleston, A. G. (1951). Arch. industr. Hyg., 4, 270.

Policard, A. (1950). Wiss. ForschBer., 60, 262. Policard Collet, A. (1951). C.R. Acad. Sci., Paris, 233, 1159. 